\title{
Cepharanthine promotes the effect of dexmedetomidine on the deposition of $\beta$-amyloid in the old age of the senile dementia rat model by regulating inflammasome expression
}

Youhua Wu, Yunfei Cao, Shuyu Wang, Xianfei Xu, Ming Wang

Department of Anaesthesiology, People's Hospital of Beilun District, Ningbo City, Zhejiang Province, Ningbo, Zhejiang, China

\begin{abstract}
Aim of the study: The present study evaluates the synergistic effect of cepharanthine $(C P)$ with dexmedetomidine (DEM) on the deposition of $\beta$-amyloid $(A \beta)$ in the brain tissue of senile dementia $(S D)$ rats.

Material and methods: Senile dementia was induced by injecting D-gal intraperitoneally $60 \mathrm{mg} / \mathrm{kg} /$ day for six weeks and Aß1-42 $(5 \mu \mathrm{ll}$ ) intracranially. The effect of cepharanthine and dexmedetomidine was estimated by determining the cognitive function and neurological function score. Moreover, mediators of inflammation, parameters of oxidative stress and reactive oxygen species (ROS) were determined in the brain tissue of senile dementia rats. Mitochondrial membrane permeability and deposition of A $A 1-42$ was estimated in senile dementia rats. Western blot assay and reverse transcription polymerase chain reaction (RT-PCR) was performed for the expression of proteins and genes in the brain tissue of senile dementia rats.

Results: Data of the study reveal that cepharanthine alone and in combination with dexmedetomidine improves the neurological function score and cognitive function in SD rats. Moreover, parameters of oxidative stress, inflammatory mediators and production of ROS in CP, DEM and CP + DEM treated groups were compared to the SD group of rats. Treatment with $C P, D E M$ and $C P+D E M$ ameliorates the altered expression of NLRP3 pathway and deposition of $A \beta$ in the brain tissue of SD rats.

Conclusions: In conclusion, data reveal that cepharanthine ameliorates the deposition of A $\beta$ and NLRP3 pathway in $S D$ rats. Moreover, cepharanthine treatment with dexmedetomidine shows the synergistic effect against the aged $S D$ rat model.
\end{abstract}

Key words: cepharanthine, dexmedetomidine, $\beta$-amyloid, dementia, inflammasomes.

\section{Introduction}

Senile dementia (SD) is a neurodegenerative disorder which commonly occurs in the old age, is characterized by cognitive dysfunction and loss of memory, which affects common daily functioning of patients [1]. A report reveals that $7 \%$ of people aged 65 suffer from dementia in China $[20,21]$. SD is one of the types of dementia commonly associated with Alzheimer's disease (AD). There are several pathogeneses which contribute to the development $A D$ including deposition of $\beta$-amyloid [13]. The literature reveals that deposition of $\beta$-amyloid alters the permeability of cell membranes of the neurons, induces the production of reactive oxygen species and activates the inflammasomes [8]. These factors cause apoptosis of neuronal cells. The reported study 
reveals that inhibition of NLRP3 ameliorates the formation of $\beta$-amyloid plaques and degeneration of neurons [7]. Conventional treatment available for the management of $A D$ and the recent years' alternative medicine has shown therapeutic potential for the treatment of chronic disorders.

Cepharanthine (CP) is an alkaloid isolated from Stephania cepharantha Hayata [9]. The literature reveals that $C P$ has shown a potential effect against several types of cancer such as nasopharyngeal carcinoma, cervical adenocarcinoma, osteosarcoma, cholangiocarcinoma, hepatocellular carcinoma, leukemia and oral squamous cell carcinoma $[11,15]$. Cepharanthine is also reported for its anti-inflammatory, liver injury, diabetic nephropathy, spinal cord injury and renal injury activity $[2,14,16]$. Cepharanthine was found to be inhibiting the NF- $\mathrm{KB}$ and NLRP3 inflammasome and thereby to protect from diabetic nephropathy [16]. Thus, the present study determines the protective effect of cepharanthine and its synergistic effect with dexmedetomidine (DEM) on the deposition of $\beta$-amyloid in a rat model of dementia.

\section{Material and methods}

\section{Animals}

Male SD rats of 200-230 g weight were kept under the standard conditions (humidity: $60 \pm 5 \%$; temperature: $24 \pm 3^{\circ} \mathrm{C}$ ) for $12 \mathrm{~h}$ light and dark cycle. All the protocols of the study were approved by the institutional animal ethical committee by People's Hospital of Beilun District, China (IAEC/PH-BD/2017/05).

\section{Chemicals}

Cepharanthine and DEX were procured from Sigma Aldrich, USA. ELISA kits for the estimation of mediators of inflammation and parameters of oxidative stress were purchased from R\&D Systems, USA. The RevertAid First Strand cDNA Synthesis kit was purchased from Thermo Scientific, USA. Antibodies used in western blot assay were purchased from Santa Cruz, USA.

\section{Experiments}

Fifty rats were separated into five groups $(n=10)$ such as the control group, SD group, CP group receiving cepharanthine $10 \mathrm{mg} / \mathrm{kg}$, DEM group receiving dexmedetomidine $40 \mathrm{mg} / \mathrm{kg}$ and $\mathrm{CP}+\mathrm{DEM}$ group receiving cepharanthine $10 \mathrm{mg} / \mathrm{kg}$ and dexmedetomidine $40 \mathrm{mg} / \mathrm{kg}$ for the same period.
Senile dementia was induced in rats as per previously reported study [18]. The aging model was induced in all the animals by injecting D-gal intraperitoneally $60 \mathrm{mg} / \mathrm{kg} /$ day for the duration of six weeks and control group rats received the saline solution for the same period. All the animals were anesthetized by injecting $80 \mathrm{mg} / \mathrm{kg}$ pentobarbital intraperitoneally and rats were placed on the stereotaxic apparatus and at the right ventricle $(3.5 \mathrm{~mm}$ under cortical surface, $1.5 \mathrm{~mm}$ right to raphe and $1.0 \mathrm{~mm}$ behind anterior fontanelle) cannulas were fixed after drilling. A $31-42(5 \mu \mathrm{l})$ of $2 \mu \mathrm{g} / \mu \mathrm{l}$ concentration was slowly injected directly in the brain. Later on animals were treated as per the respective group by gastric gavage for one more week.

\section{Determination of behavioural changes}

The Morris water maze (MWM) was used to determine behavioural changes as per the previously reported study [20]. The water maze had the height and diameter of 40 and $100 \mathrm{~cm}$, respectively, and the platform depth was $15.5 \mathrm{~cm}$. Four quadrants were created in the apparatus with a thread and one quadrant of the platform was placed so that it could not be visualised. Swimming behaviour was monitored continuously for 6 days. Escape latency was determined as an indicator of spatial memory and the effect of CP was estimated on the vascular dementia.

\section{Estimation of the neurological function}

The modified neurological severity score (mNSS) was used to determine balance, touch, vision, abnormal behaviour, muscle mass, sensation and motion, as previously reported. The mNSS was calculated on a 0-18 scale, where 0 indicated a normal brain function and 18 indicated a severe deficit. Neurological function was determined at the end of the protocol.

\section{Biochemical parameters}

ELISA kits were used to assess the mediators of inflammation in the brain tissue (nuclear factor $\kappa B-$ NF- $\kappa B$, interleukin (IL)- 6 and IL-18) of SD rats as per the manufacturer's directions. Moreover, oxidative stress markers, such as the level of malondialdehyde (MDA) and activity of glutathione peroxidase (GPX) and super oxide dismutase (SOD), were measured in the brain tissue using ELISA kits. 


\section{Western blot assay}

Protein samples were isolated from homogenised tissues and quantified using a bicinchoninic acid (BCA) assay kit. We used $10 \%$ sodium dodecyl sulphate-polyacrylamide gel electrophoresis (SDSPAGE) to separate the proteins. Proteins were then transferred to a nitrocellulose membrane using an electroblotting technique. The membrane was blocked using a 5\% blocking solution (non-fat milk) and then incubated in a blocking buffer with the following primary antibodies overnight at $4^{\circ} \mathrm{C}$ : ASC (dilution of 1 : 100), p20 (dilution of $1: 500$ ), NLRP3 (dilution of 1 : 100), caspase-3 (dilution of 1 : 200) and $\beta$-actin (dilution of 1 : 100). The following day, goat secondary antibody conjugated with horseradish peroxidase (HRP) was added to the blocking buffer (1 : 1,000, non-fat milk), and a chemiluminescence kit (Thermo Fisher Scientific, Shanghai, China) was used to detect the proteins.

\section{Reverse-transcription polymerase chain reaction (RT-PCR)}

RNA was isolated from separated brain tissue using TRIzol Reagent (Thermo Fisher). The RevertAid First Strand cDNA Synthesis Kit (Thermo Fisher) was used to reverse-transcribe the RNA. Specific primers were mixed with RT-2 SYBR green master mix to evaluate gene expression by RT-PCR. The procedure used for all samples was as follows: $98^{\circ} \mathrm{C}$ for $2 \mathrm{~min}$, followed by $25-40$ cycles of $98^{\circ} \mathrm{C}$ for $10 \mathrm{~s}$, then $55^{\circ} \mathrm{C}$ for $5 \mathrm{~s}$, and $72^{\circ} \mathrm{C}$ for 20 s. mRNA expression levels of P2X4 and 18S rRNA were calculated according to relative standard curves, which were generated by plotting the quantification cycle $(\mathrm{Cq})$ against the log amount of total cDNA added to the reaction. $2^{-\Delta \Delta C q}$ method was used to estimate the expression of the relative target genes.

$\begin{array}{lll}\text { Primers Forward } & \text { Backward } \\ \text { NLRP3 } & \text { 5'-GCTAAGAAGGACCAGCCAGAGT -3' 5'-GAACCTGCTTCTCACATGTCGT-3' } \\ \text { ASC } & \text { 5'-TGCTTAGAGACATGGGCTTAC-3' } & \text { 5'-CTGTCCTTCAGTCAGCACACT-3' } \\ \text { IL-18 } & \text { 5'-AGTGAACCCCAGACCAGACT-3' } & \text { 5'-TCCTTCACAGAGAGGGTCACA-3' } \\ \text { NF-KB } & \text { 5'-GAGCAAATGGTGAAGGAG-3' } & \text { 5'-TCTGGAAGTTGAGGAAGG-3' } \\ \text { GAPDH } & \text { 5'-AACTTTGGCATTGTGGAAGG-3' } & \text { 5'-GGATGCAGGGATGATGTTCT-3' }\end{array}$

\section{Estimation of reactive oxygen species}

Brain tissue homogenate $(50 \mu \mathrm{l})$ was mixed with potassium phosphate buffer $(4.85 \mathrm{ml})$ and the mixture was incubated after adding 2',7'-dichloro- fluorescindiacetate dye at $37^{\circ} \mathrm{C}$ for $15 \mathrm{~min}$. Then the mixture was centrifuged at $12000 \times\left(4^{\circ} \mathrm{C}\right)$ for $10 \mathrm{~min}$ and $5 \mathrm{ml}$ of $100 \mathrm{mmol} /$ LPBS on a vortex was added to the mixture at $0^{\circ} \mathrm{C}$ and further incubated at $37^{\circ} \mathrm{C}$ for $60 \mathrm{~min}$. Absorbance was estimated at $488 \mathrm{~nm}$ for excitation and $525 \mathrm{~nm}$ for emission.

\section{Determination of the membrane potential of mitochondria}

Mitochondrial membrane potential was estimated by using lipophilic cationic fluorescent dye 5,5',6,6'-terachloro-1,1',3,3'-tetraethylbenzimidazolcarbocyanine iodide (JC-1). Osteoblast cells $\left(2 \times 10^{5}\right.$ cells/well) were isolated in a 6-well plate and Mitochondrial Membrane Potential Detection Kit was used to perform the estimation of MMP as per the manufacturer's instructions.

\section{Immunohistochemical staining}

The brain was isolated from each animal and placed in paraffin after dehydrating the brain tissue. Brain tissue was sectioned using microtome and further incubated with mouse anti-human amyloid protein 17-24 monoclonal antibody at $4{ }^{\circ} \mathrm{C}$ overnight. Vectastain kit with $0.05 \%$ diaminobenzidine was used to develop the immunolabeled sections. The image analyser was used to observe the changes in the brain tissue section.

\section{Statistical analysis}

All data are expressed as means \pm standard error of the mean (SEM; $n=10)$ and the statistical analysis consisted of a one-way analysis of variance (ANOVA). Post-hoc comparisons of means were carried out with Dunnett's post hoc test using GraphPad Prism software (ver. 6.1; San Diego, CA, USA). $P$ values $<0.05$ were considered to indicate statistical significance.

\section{Results}

\section{Cepharanthine alone and in combination with dexmedetomidine ameliorates the cognitive function}

In senile dementia rats treated with cepharanthine alone and in combination with dexmedetomidine, the cognitive function was estimated by MWM. There was a decrease in the number of crossings and time spent in the target quadrant and increase in the escape latency in the SD group compared to the control group of rats. However, treatment with cepharanthine and dex- 
medetomidine alone and in combination ameliorates the altered cognitive function in senile dementia rats. It was also observed that the number of crossings and time spent in the target quadrant was enhanced significantly $(p<0.01)$ and the escape latency decreased in the CP + DEM treated group compared to the CP and DEM alone treated group (Fig. 1).

\section{Cepharanthine alone and in combination with dexmedetomidine ameliorates neurological functions}

The neurological function score was determined in senile dementia rats treated with cepharanthine alone and in combination with dexmedetomidine (Fig. 2). In the SD group of rats, the neurological function score was enhanced significantly ( $p<$ 0.01) compared to the control group. There was a decrease in the neurological function score in $C P$, $D E M$ and $C P+D E M$ treated groups compared to the SD group of rats. However, the CP + DEM group shows further a significant reduction in the neurological function score compared to CP and DEM alone treated groups.

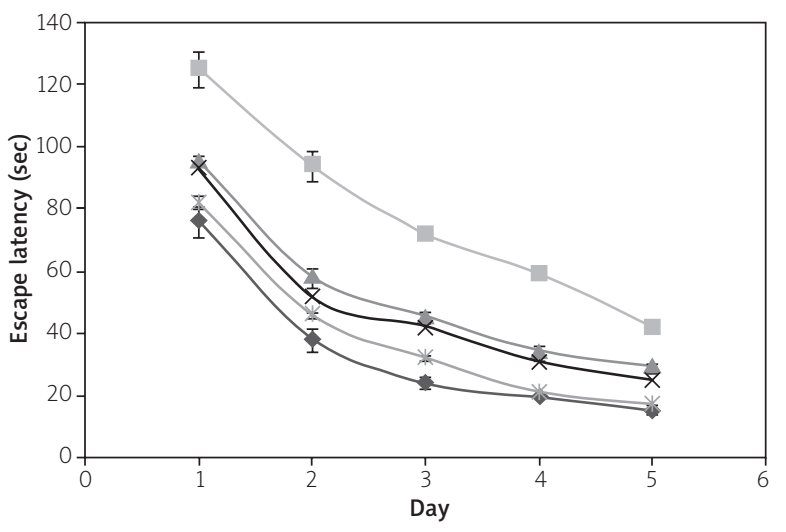

$$
\begin{aligned}
& \rightarrow \text { Control } \\
& - \text { SD } \\
& \text { - CP } \\
& \text { * DEM } \\
& * \text { CP + DEM }
\end{aligned}
$$

Fig. 1. Effect of cepharanthine alone and in combination with dexmedetomidine attenuates the cognitive dysfunction in the senile dementia rat model. Mean \pm SEM $(n=10)$; ${ }^{@} p<0.01$ compared to the control group; ${ }^{\star \star} p<0.01$ compared

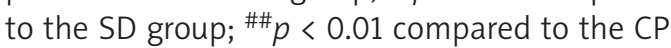
and DEM group.

\section{Effect of cepharanthine alone and in combination with dexmedetomidine on the mediators of inflammation}

The effect of cepharanthine alone and in combination with dexmedetomidine on the mediators of inflammation in the brain tissue of senile dementia rats was determined (Fig. 3). The level of mediators of inflammation such as IL-6, IL-18 and NF- $\kappa$ B was significantly $(p<0.01)$ enhanced in the brain tissue of the SD group compared to the control group of rats. There was a reduction in the level of mediators of inflammation in CP, DEM and CP + DEM treated groups compared to the $\mathrm{SD}$ group of rats. However, mediators of inflammation were reduced further in the $C P+D E M$ treated group compared to the CP and DEM treated group of rats.

\section{Effect of cepharanthine alone and in combination with dexmedetomidine on the parameters of oxidative stress and production of reactive oxygen species}

The effect of cepharanthine alone and in combination with dexmedetomidine on the parameters of
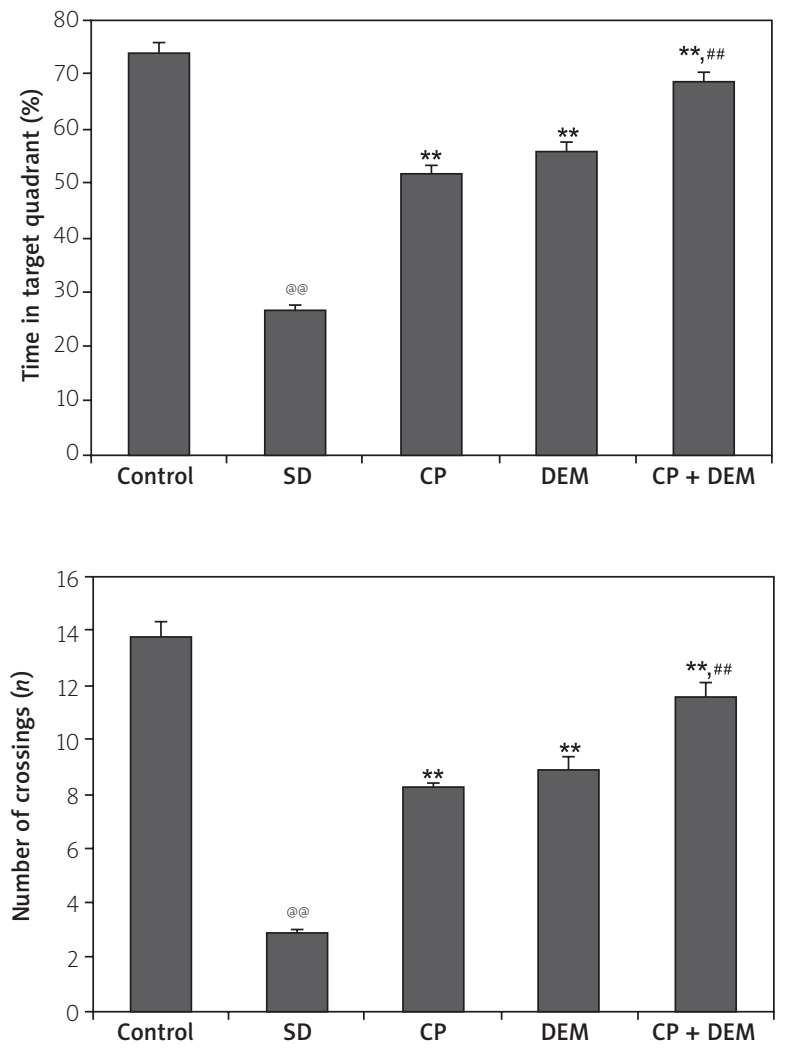


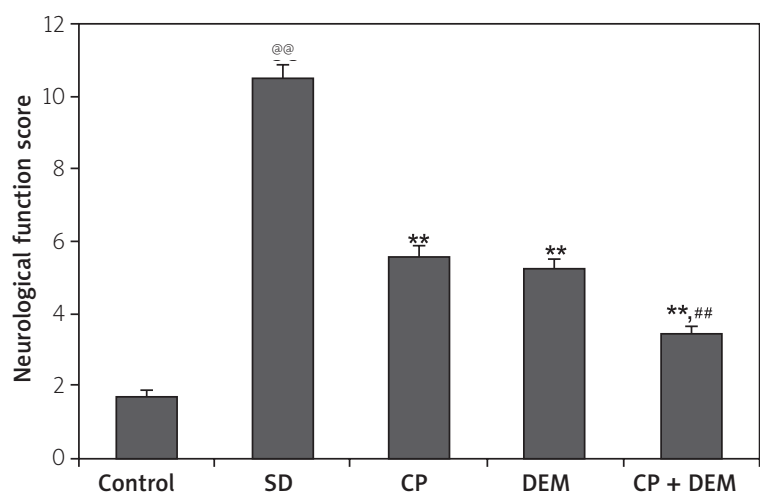

Fig. 2. Effect of cepharanthine alone and in combination with dexmedetomidine attenuates the neurological function score in the senile dementia rat model. Mean \pm SEM $(n=10)$; ${ }^{\circledR} p<0.01$ compared to the control group; ${ }^{* *} p<0.01 \mathrm{com}$ pared to the SD group; ${ }^{\# \#} p<0.01$ compared to the $\mathrm{CP}$ and DEM group.

oxidative stress and production of reactive oxygen species in the brain tissue of senile dementia rats was estimated. The level of MDA was enhanced and activity of SOD was reduced in the SD group compared to the control group of rats. There was a significant $(p<0.01)$ reduction in the level of MDA and increase in the activity of SOD in the brain tissue of CP, DEM and CP + DEM treated groups compared to the SD group of rats. Moreover, treatment with $C P, D E M$ and CP + DEM ameliorates the enhanced production of ROS in the brain tissue of SD rats. However, treatment with CP + DEM significantly reduced the level of MDA and production of ROS and increased the activity of SOD compared to the CP and DEM treated group of rats (Fig. 4).

\section{Effect of cepharanthine alone and in combination with dexmedetomidine on the expression of ASC, NLRP3, caspase- 3 and $\mathrm{p} 20$ protein}

The expression of ASC, NLRP3, caspase- 3 and p20 protein was estimated in the brain tissue of senile dementia rats treated with cepharanthine alone and in combination with dexmedetomidine (Fig. 5). There was an increase in the expression of ASC, NLRP3, caspase-3 and p20 protein in the brain tissue of the SD group compared to the control group of rats. It was observed that expression of ASC, NLRP3,

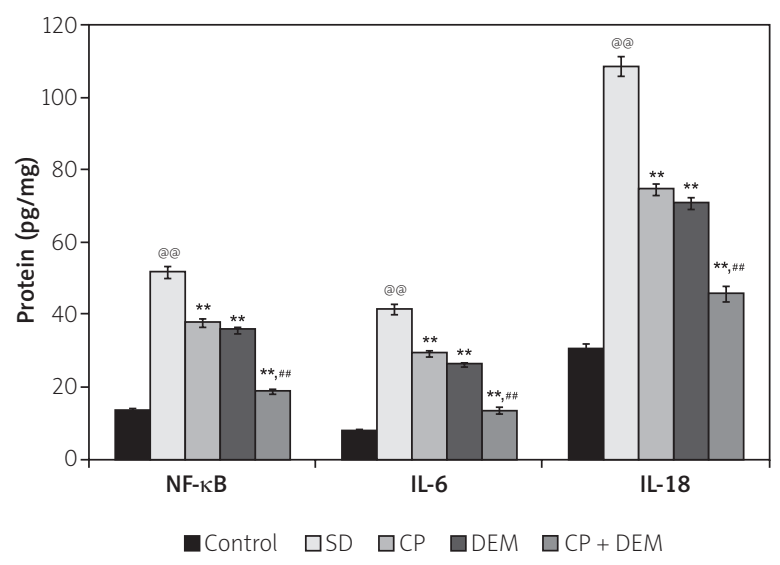

Fig. 3. Effect of cepharanthine alone and in combination with dexmedetomidine attenuates the mediators of inflammation in the brain tissue of senile dementia rats. Mean \pm SEM $(n=10)$; ${ }^{\circledR} p<0.01$ compared to the control group; ${ }^{* *} p<0.01$ compared to the SD group; ${ }^{\# \#} p<0.01$ compared to the CP and DEM group.

caspase- 3 and p20 protein was reduced significantly $(p<0.01)$ in groups treated with cepharanthine alone and in combination with dexmedetomidine compared to the SD group of rats. CP treatment in combination with DEM significantly $(p<0.01)$ reduces the expression of ASC, NLRP3, caspase-3 and p20 protein compared to the CP and DEM alone treated group of rats.

\section{Effect of cepharanthine alone and in combination with dexmedetomidine on the mRNA expression of NLRP3, NF- $\kappa B, I L-18$ and ASC}

mRNA expression of NLRP3, NF- $\kappa$ B, IL-18 and ASC was determined in the brain tissue of CP, $D E M$ and $C P+D E M$ treated SD rats. There was an increase in the mRNA expression of NLRP3, NF- $\kappa B$, IL-18 and ASC in the brain tissue of the SD group compared to the control group of rats. It was observed that mRNA expression of NLRP3, NF- $\kappa B$, IL-18 and ASC was reduced significantly $(p<0.01)$ in groups treated with cepharanthine alone and in combination with dexmedetomidine compared to the SD group of rats. CP treatment in combination with DEM significantly $(p<0.01)$ reduces the mRNA expression of NLRP3, NF- $\kappa \mathrm{B}, \mathrm{IL}-18$ and ASC compared to the $\mathrm{CP}$ and $\mathrm{DEM}$ alone treated group of rats (Fig. 6 ). 


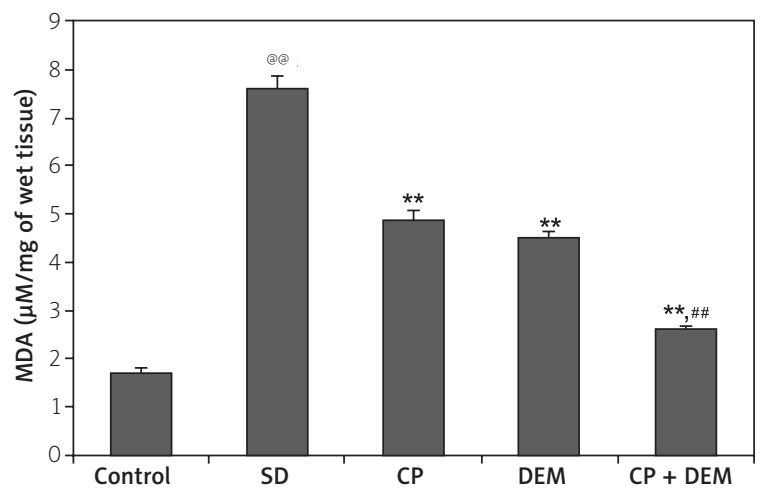

Fig. 4. Effect of cepharanthine alone and in combination with dexmedetomidine attenuates the parameters of oxidative stress and production of reactive oxygen species in the brain tissue of senile dementia rats. Mean $\pm \operatorname{SEM}(n=10)$; ${ }^{\circledR} p<0.01$ compared to the control group; ${ }^{* *} p<0.01$ compared to the SD group; ${ }^{\# \# p<0.01}$ compared to the CP and DEM group.

\section{Effect of cepharanthine alone and in combination with dexmedetomidine on the MMP}

Figure 7 shows the changes in the MMP of groups treated with cepharanthine alone and in combination with dexmedetomidine in the brain tissue of senile dementia rats. There was a decrease in the MMP in the brain tissue of the SD group compared to the control group of rats. MMP was enhanced in the brain tissue of CP, DEM and CP + DEM treated groups compared to the SD group of rats. However, the CP + DEM group showed a significantly enhanced MMP compared to the CP and DEM alone treated group of rats.

\section{Effect of cepharanthine alone and in combination with dexmedetomidine on the deposition of $A \beta 1-42$ protein}

The effect of cepharanthine alone and in combination with dexmedetomidine on the deposition of $A \beta 1-42$ in the brain tissue of senile dementia rats was determined as shown in Figure 8. It was observed that the ratio of A $\beta 1-42$ aggregation was enhanced in the SD group compared to the control group of rats. Treatment with CP and DEM alone and
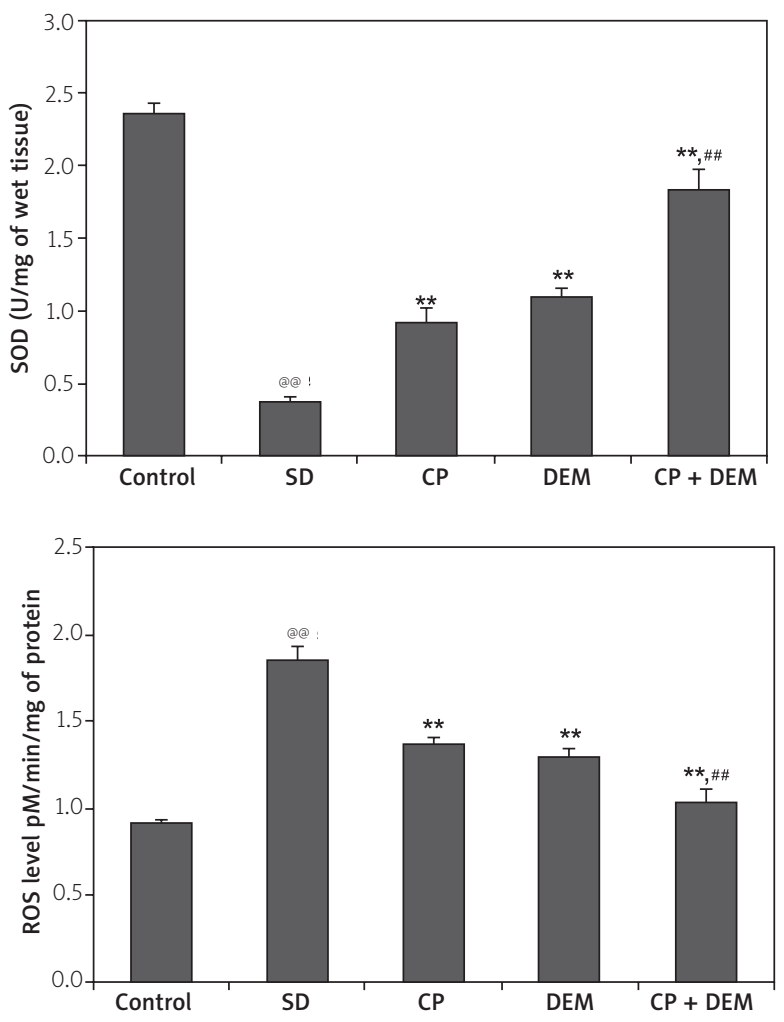

in combination ameliorates the enhanced ratio of A $\beta 1-42$ aggregation in the brain tissue of SD rats. However, the ratio of $A \beta 1-42$ aggregation was significantly reduced $(p<0.01)$ in the brain tissue of the $C P+D E M$ treated group compared to the CP and DEM group of rats.

\section{Discussion}

Senile dementia is a neurodegenerative disorder commonly associated with AD and its management still poses a challenge. The present study evaluates the synergistic effect of cepharanthine with dexmedetomidine on the deposition of beta amyloid in the brain tissue of senile dementia rats. The effect of cepharanthine alone and in combination with dexmedetomidine on senile dementia was estimated by determining the cognitive function using MWM and neurological function score. Moreover, mediators of inflammation, parameters of oxidative stress and ROS were determined in the brain tissue of senile dementia rats. Mitochondrial membrane permeability and deposition of $A \beta 1-42$ was estimated in the brain tissue of senile dementia rats. Western blot assay and RT-PCR was performed for the expression 

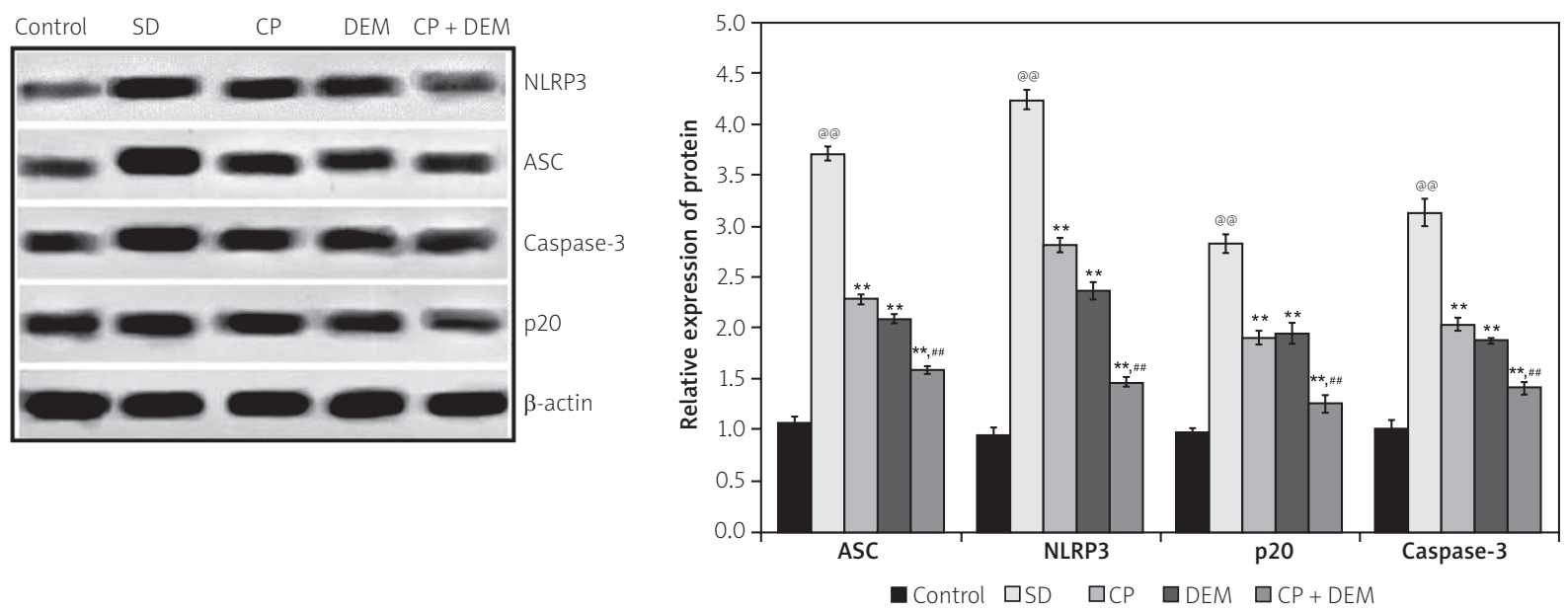

Fig. 5. Effect of cepharanthine alone and in combination with dexmedetomidine attenuates the expression of ASC, NLRP3, caspase-3 and p20 protein in the brain tissue of senile dementia rats. Mean \pm SEM $(n=10)$; @@ $p<0.01$ compared to the control group; ${ }^{* \star} p<0.01$ compared to the SD group; ${ }^{\# \#} p<0.01$ compared to the CP and DEM group.

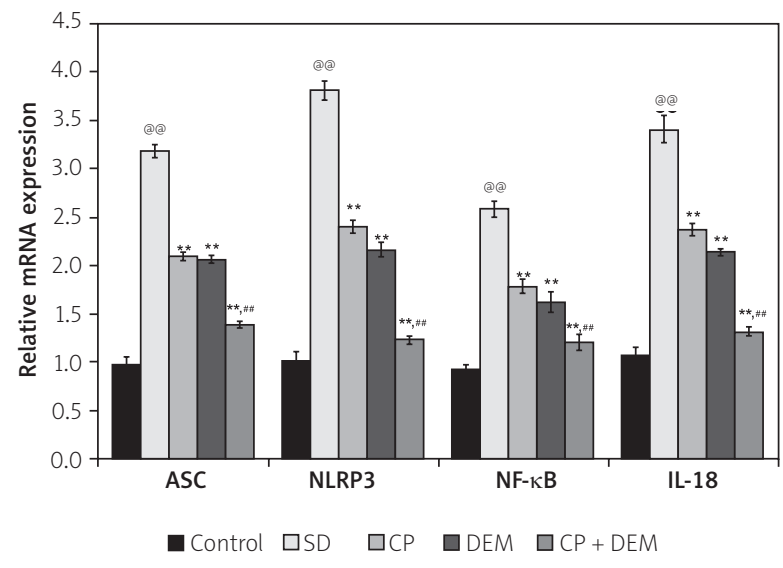

Fig. 6. Effect of cepharanthine alone and in combination with dexmedetomidine attenuates the mRNA expression of NLRP3, NF- $\kappa \mathrm{B}, \mathrm{IL}-18$ and ASC in the brain tissue of senile dementia rats. Mean \pm SEM $(n=10)$; @ $p<0.01$ compared to the control group; ${ }^{* *} p<0.01$ compared to the SD group; $\# p<0.01$ compared to the CP and DEM group.

of proteins and genes in the brain tissue of senile dementia rats.

The aged animal model of $A D$ resembles the pathophysiology and morphological characteristics of human AD [19]. Chronic D-gal administration produces cognitive dysfunction and neurodegeneration similar to that in aged rats and thus has a well proved AD rat model [4]. Moreover, AD was characterized histopathologically by formation of

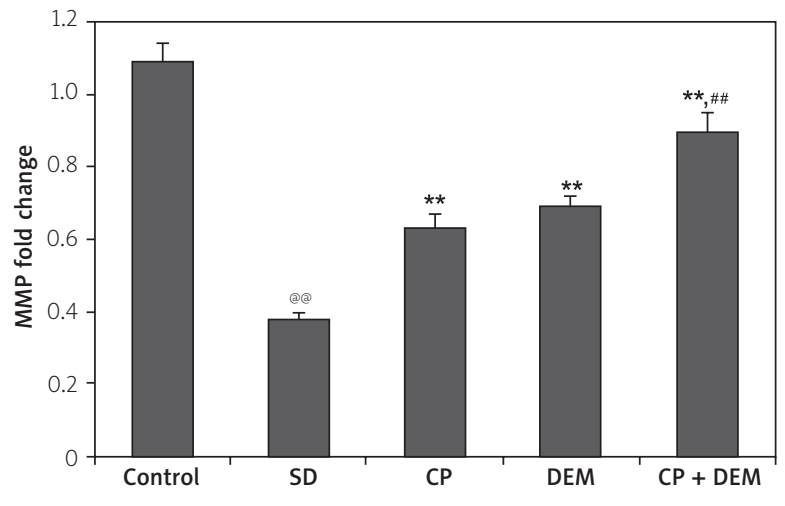

Fig. 7. Effect of cepharanthine alone and in combination with dexmedetomidine attenuates the changes in the MMP in the brain tissue of senile dementia rats. Mean \pm SEM $(n=10)$; @@ $p<0.01$ compared to the control group; ${ }^{* *} p<0.01$ compared to the SD group; ${ }^{\# \#} p<0.01$ compared to the CP and DEM group.

$A \beta$ plaques in the brain tissue and intracerebroventricular administration of $A \beta$ in rats produces $A D$ or dementia [12]. Thus, in the present investigation SD was induced by chronic administration of D-gal and $A \beta 1-42$ in a rat model. The literature reveals that deposition of $A \beta 1-42$ induces neurodegeneration by an increase in oxidative stress, activation of the inflammatory pathway and stimulating the apoptosis by the caspase pathway [17]. 

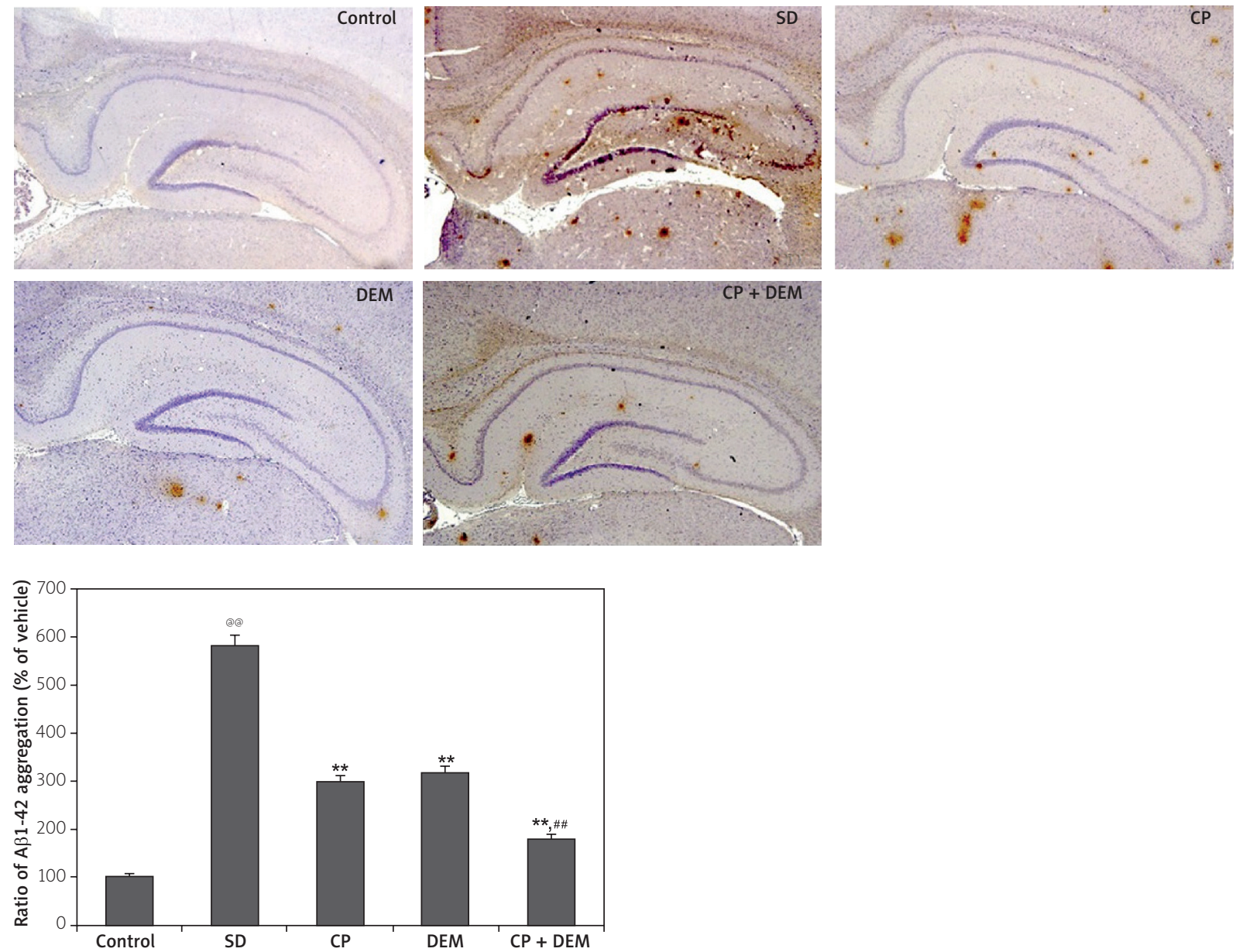

Fig. 8. Effect of cepharanthine alone and in combination with dexmedetomidine on the deposition of A $\beta 1-42$ in the brain tissue of senile dementia rats. Mean \pm SEM $(n=10)$; ${ }^{\circledR} p<0.01$ compared to the control group; ${ }^{* *} p<0.01$ compared to the SD group; ${ }^{\# \#} p<0.01$ compared to the CP and DEM group.

$\beta$-amyloid is reported to enhance the production of ROS and thereby induces oxidative stress [3]. This enhanced oxidative stress alters the permeability of the cell and mitochondrial membrane, which leads to activation of the apoptosis pathway. Natural antioxidants have shown a beneficial effect on the cellular apoptosis and thus antioxidants are explored as an AD treatment. The literature has suggested that dexmedetomidine has strong antioxidant and anti-inflammatory properties [10]. Data of the present investigation suggest that cepharanthine alone and in combination with dexmedetomidine ameliorates the altered level of oxidative stress and production of ROS and MMP in SD rats.

Alzheimer's disease is also recognized as a neuroinflammatory disorder and several mediators of inflammation like NF-אB, IL-6 and IL-18 are reported to be enhanced in $A D$, which contributes to the neurodegeneration [6]. Cepharanthine has shown potential anti-inflammatory properties [16] and data of the study suggest that cepharanthine shows a synergistic effect with dexmedetomidine on mediators of inflammation in the brain tissue of SD rats. Moreover, NLRP3 inflammasome is activated due to increased production of ROS in AD patients [22]. NLRP3 activates the caspase pathway and thereby induces apoptosis of neuronal cells [5]. Results of the investigation reported that the treatment with cepharanthine alone and in combination with dexmedetomidine ameliorates the altered expression of NLRP3, ASC, p20 and caspase-3 protein in the brain tissue of SD rats.

In addition, treatment with cepharanthine alone and in combination with dexmedetomidine reduces 
the deposition of $A \beta 1-42$ in the brain tissue compared to the SD group of rats. Data suggest that cepharanthine shows a potential effect when given with dexmedetomidine for the management of $A D /$ senile dementia.

\section{Conclusions}

In conclusion, data reveal that cepharanthine ameliorates the deposition of beta amyloid and NLRP3 pathway in SD rats. Moreover, cepharanthine treatment in combination with dexmedetomidine shows a synergistic effect against the aged SD rat model.

\section{Acknowledgements}

Authors of this manuscript are thankful to Zhejiang TCM science and technology project (No: 2019ZA120), and Ningbo medical science and technology project of Zhejiang Province (No: 2018A08) for providing funding to support the presented work.

\section{Disclosure}

The authors report no conflict of interest.

\section{References}

1. Apostolova LG. Alzheimer disease. Continuum (Minneap Minn) 2016; 22 (2 Dementia): 419-434.

2. Chang YK, Huang SC, Kao MC, Huang CJ. Cepharanthine alleviates liver injury in a rodent model of limb ischemia-reperfusion. Acta Anaesthesiol Taiwan 2016; 54: 11-15.

3. Cheignon C, Tomas M, Bonnefont-Rousselot D, Faller P, Hureau C, Collin F. Oxidative stress and the amyloid beta peptide in Alzheimer's disease. Redox Biol 2018; 14: 450-464.

4. Chiroma SM, Baharuldin MTH, Mat Taib CN, Amom Z, Jagadeesan S, Ilham Adenan M, Mahdi O, Moklas MAM. Centella asiatica protects d-galactose/AlCl 3 mediated Alzheimer's disease-like rats via PP2A/GSK-3 $\beta$ signaling pathway in their hippocampus. Int J Mol Sci 2019; 20: 1871.

5. Choi AJ, Ryter SW. Inflammasomes: molecular regulation and implications for metabolic and cognitive diseases. Mol Cells 2014; 37: 441-448.

6. Glass CK, Saijo K, Winner B, Marchetto MC, Gage FH. Mechanisms underlying inflammation in neurodegeneration. Cell 2010; 140: 918-934.

7. Gold M, El Khoury J. $\beta$-amyloid, microglia, and the inflammasome in Alzheimer's disease. Semin Immunopathol 2015; 37: 607-611.

8. Hohsfield LA, Humpel C. Migration of blood cells to $\beta$-amyloid plaques in Alzheimer's disease. Exp Gerontol 2015; 65: 8-15.

9. Huang H, Hu G, Wang C, Xu H, Chen X, Qian A. Cepharanthine, an alkaloid from Stephania cepharantha Hayata, inhibits the inflammatory response in the RAW264.7 cell and mouse models. Inflammation 2014; 37: 235-246.
10. Jiang Y, Xia M, Huang Q, Ding D, Li Y, Zhang Z, Zhang X. Protective effect of dexmedetomidine against organ dysfunction in a two-hit model of hemorrhage/resuscitation and endotoxemia in rats. Braz J Med Biol Res 2019; 52: e7905.

11. Katanyoo K, Sanguanrungsirikul S, Manusirivithaya S. Comparison of treatment outcomes between squamous cell carcinoma and adenocarcinoma in locally advanced cervical cancer. Gynecol Oncol 2012; 125: 292-296.

12. Lecanu L, Papadopoulos V. Modeling Alzheimer's disease with non-transgenic rat models. Alzheimers Res Ther 2013; 5: 17.

13. Murphy MP, LeVine $\mathrm{H}$ 3rd. Alzheimer's disease and the amyloid-beta peptide. J Alzheimers Dis 2010; 19: 311-323.

14. Nagano M, Kanno T, Fujita H, Muranaka S, Fujiwara T, Utsumi K. Cepharanthine, an anti-inflammatory drug, suppresses mitochondrial membrane permeability transition. Physiol Chem Phys Med NMR 2003; 35: 131-143.

15. Rattanawong A, Payon V, Limpanasittikul W, Boonkrai C, Mutirangura A, Wonganan $\mathrm{P}$. Cepharanthine exhibits a potent anticancer activity in p53-mutated colorectal cancer cells through upregulation of p21Waf1/Cip1. Oncol Rep 2018; 39: 227-238.

16. Samra YA, Said HS, Elsherbiny NM, Liou GI, El-Shishtawy MM, Eissa LA. Cepharanthine and Piperine ameliorate diabetic nephropathy in rats: role of NF-KB and NLRP3 inflammasome. Life Sci 2016; 157: 187-199.

17. Solleiro-Villavicencio H, Rivas-Arancibia S. Effect of chronic oxidative stress on neuroinflammatory response mediated by $\mathrm{CD}^{+} \mathrm{T}$ cells in neurodegenerative diseases. Front Cell Neurosci 2018; 12: 114.

18. Thomé GR, Oliveira VA, Chitolina Schetinger MR, Saraiva RA, Souza D, Dorneles Rodrigues OE, Teixeira Rocha JB, Ineu RP, Pereira ME. Selenothymidine protects against biochemical and behavioral alterations induced by ICV-STZ model of dementia in mice. Chem Biol Interact 2018; 294: 135-143.

19. Vickers JC, Mitew S, Woodhouse A, Fernandez-Martos CM, Kirkcaldie MT, Canty AJ, McCormack GH, King AE. Defining the earliest pathological changes of Alzheimer's disease. Curr Alzheimer Res 2016; 13: 281-287.

20. Vorhees CV, Williams MT. Morris water maze: procedures for assessing spatial and related forms of learning and memory. Nat Protoc 2006; 1: 848-858.

21. Xu J, Wang J, Wimo A, Fratiglioni L, Qiu C. The economic burden of dementia in China, 1990-2030: implications for health policy. Bull World Health Organ 2017; 95: 18-26.

22. Wei P, Yang F, Zheng Q, Tang W, Li J. The potential role of the NLRP3 inflammasome activation as a link between mitochondria ROS generation and neuroinflammation in postoperative cognitive dysfunction. Front Cell Neurosci 2019; 13: 73. 